\title{
Parallel Matched Filtering Algorithm with Low Complexity
}

\author{
Cheng Xuhuang ${ }^{1, a}$, Shao Gaoping ${ }^{1, \mathrm{~b}}$ and Wang Yang ${ }^{1, \mathrm{c}}$ \\ ${ }^{1}$ College of Information System Engineering, Information Engineering University, China \\ a498532715@qq.com, ${ }^{\mathrm{b}} 572798566 @ q q . c o m,{ }^{\mathrm{c} w y w y 79 @ 163 . c o m ~}$
}

Keywords: overlap-save; low complexity; parallel matched filter; high speed demodulation.

\begin{abstract}
For the problem of double counting in the overlap save algorithm(OSA), this paper presents parallel matched filtering algorithm with low complexity. The current data is segment based on the filter order, then using the quarter discrete fourier transform (QDFT) to reduce the amount of calculation. The calculation result of the previous and current data block are added to obtain the block filter results. Analysis and simulation results show that the algorithm effectively reduces the computational complexity. It is more suitable for high-speed demodulation which has multiple parallel paths.
\end{abstract}

\section{Introduction}

With the rapid development of information technology and communication technology, the data transmission rate of communication system can reach the order of Gsps. High-speed data can not be processed by traditional serial demodulation method at present, so it needs parallel processing algorithm[1] . Matched filter is high complexity part of the key algorithm, so It is significant to study the parallel matching filter algorithm.

To reduce the complexity of filtering, in [1] the complexity of the filter is reduced by optimizing the design of constant coefficient multiplier. In [1], the fast FIR algorithm (FFAs) is used to generate the low complexity filtering algorithm by using the characteristics of the filter coefficient symmetry.

In this paper, a low complexity parallel filtering algorithm is proposed on the basis of overlapped preserving filtering algorithm. The overlap-preserving algorithm is decomposed into two independent processing modules, and the low-complexity matched filter structure is calculated.

\section{Frequency - Domain Parallel Matching Filtering Algorithm Based on QDFT}

\subsection{Low complexity of the frequency domain filtering algorithm.}

Overlapping preserving method, the successive input section overlaps the data part will bring the duplicate computation, eliminate the duplication computation part, and reduce the algorithm the complexity. Through deduction, the traditional overlapping reservation method can be decomposed into two parts of the previous data block and the current data block for independent processing. The processing result of the previous data block is reserved by the last segment, and only the current data block is processed. The length of the data that needs to be involved in the calculation is reduced by half .Observing the FFT algorithm by frequency extraction (DIF), each input segment DFT result can 
be decomposed into a shorter two-part element according to the parity of its vector index.

$$
\left[\begin{array}{l}
X_{e} \\
X_{o}
\end{array}\right]=\left[\begin{array}{cc}
D_{M} & D_{M} \\
\theta_{M} & -\theta_{M}
\end{array}\right]\left[\begin{array}{l}
X_{p} \\
X_{c}
\end{array}\right]=\left[\begin{array}{c}
X_{p}^{d}+X_{c}^{d} \\
X_{p}^{\theta}-X_{c}^{\theta}
\end{array}\right]
$$

Where the elements $X_{e}$ and $X_{o}$ represents the even and odd vector indices of $X^{d}, \theta_{K}$ are Kth order odd discrete Fourier transform (ODFT) matrices,and the (n, k) elements in the matrix are $W_{K}^{n(k+1 / 2)}$.superscript $\mathrm{d}$ and $\Theta$ respectively represent the data for DFT and ODFT transform .

Divided $G^{d}$ into the same $G_{e}$ and $G_{o}$ two parts,since half of $g$ is $f$ and the rest is the M-size zero vector $0_{M}$. We have

$$
\left[\begin{array}{l}
G_{e} \\
G_{o}
\end{array}\right]=\left[\begin{array}{cc}
D_{M} & D_{M} \\
\theta_{M} & -\theta_{M}
\end{array}\right]\left[\begin{array}{c}
f \\
0_{M}
\end{array}\right]=\left[\begin{array}{l}
F^{d} \\
F^{\theta}
\end{array}\right]
$$

Also from (1) and the definitions, the circular convolution can be rewritten as:

$$
\left[\begin{array}{l}
y_{p} \\
y_{c}
\end{array}\right]=\frac{1}{2}\left[\begin{array}{c}
D_{M}^{-1}\left(F^{d} \bullet X_{p}^{d}\right)+\theta_{M}^{-1}\left(F^{\theta} \bullet X_{p}^{\theta}\right) \\
D_{M}^{-1}\left(F^{d} \bullet X_{p}^{d}\right)-\theta_{M}^{-1}\left(F^{\theta} \bullet X_{p}^{\theta}\right)
\end{array}\right]+\frac{1}{2}\left[\begin{array}{c}
D_{M}^{-1}\left(F^{d} \bullet X_{c}^{d}\right)-\theta_{M}^{-1}\left(F^{\theta} \bullet X_{c}^{\theta}\right) \\
D_{M}^{-1}\left(F^{d} \bullet X_{c}^{d}\right)+\theta_{M}^{-1}\left(F^{\theta} \bullet X_{c}^{\theta}\right)
\end{array}\right]
$$

The first half of the calculation on the right side of equation (3) relates only to $\mathrm{x}_{\mathrm{p}}$, while the latter half is only related to $\mathrm{x}_{\mathrm{c}}$. The low complexity of the frequency domain filter algorithm is shown in Figure 1, where $\mathrm{J}$ module represents the dashed part of the calculation, by the two basic modules and components. For each successive input segment, the J-module output of the current data block is calculated and added to the J-module result of the previous data, so that the result of the corresponding matched filter can be obtained and the J-module result of the current data is saved .

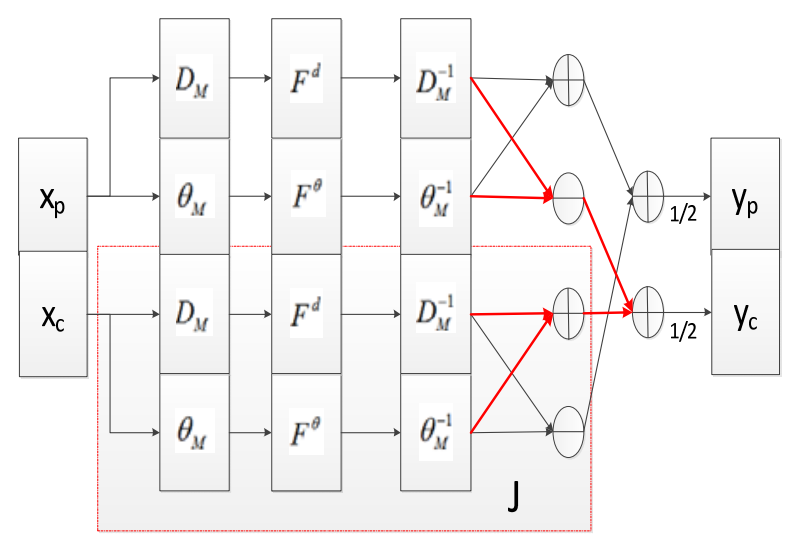

Fig.1 Schematic diagram of low-complexity frequency domain filtering algorithm

\subsection{Frequency domain matching filter algorithm based on the QDFT.}

Based on the analysis, a new filtering algorithm based on QDFT is proposed. By using the special quadruple discrete Fourier transform (QDFT) in Generalized Discrete Fourier Transform (GDFT), the $D_{2 M}^{-1}\left(G^{d} \bullet X^{d}\right)$ and $\theta_{2 M}^{-1}\left(G^{\theta} \bullet X^{\theta}\right)$ computations are simplified, and the complexity of the matched filtering algorithm is further reduced.2M point input data QDFT corresponding input and output relationship is: 


$$
X_{k}^{q}=\sum_{n=0}^{2 M-1} x_{n} W_{2 M}^{n\left(k+\frac{3}{4}\right)}
$$

QDFT Multiply the time series by the rotation factor $W_{2 M}^{(3 / 4) n}$, and then calculate the DFT results, so the same can be used FFT to quickly calculate. Through observation and analysis, in the time domain $D_{2 M}^{-1}\left(G^{d} \bullet X^{d}\right)$ can be expressed as a cyclic convolution :

$$
y_{n}=\sum_{k=0}^{n} x_{k} g_{n-k}+\sum_{k=n+1}^{2 M-1} x_{k} g_{n-k+2 M}
$$

And $\theta_{2 M}^{-1}\left(G^{\theta} \bullet X^{\theta}\right)$ corresponds to the skew circular convolution as:

$$
y_{n}=\sum_{k=0}^{n} x_{k} g_{n-k}-\sum_{k=n+1}^{2 M-1} x_{k} g_{n-k+2 M}
$$

$Q_{2 M}^{-1}\left(G^{q} \bullet X^{q}\right)$ can be expressed as a cyclic convolution formula:

$y_{n}=\sum_{k=0}^{n} x_{k} g_{n-k}+j \sum_{k=n+1}^{2 M-1} x_{k} g_{n-k+2 M}$

$Q_{K}$ are Kth order quarter discrete fourier transform (QDFT) matrices, and the (n, k) elements in the matrix are $W_{K}^{n(k+3 / 4)}$, The superscript $\mathrm{q}$ indicates that the data undergo QDFT transformation.Comparing (5) and (6) with (7), we can see that the real part of $Q_{2 M}^{-1}\left(G^{q} \bullet X^{q}\right)$ is equivalent to $\left(D_{2 M}^{-1}\left(G^{d} \bullet X^{d}\right)+\theta_{2 M}^{-1}\left(G^{\theta} \bullet X^{\theta}\right)\right) / 2$ and the imaginary part is equivalent to $\left(D_{2 M}^{-1}\left(G^{d} \bullet X^{d}\right)-\theta_{2 M}^{-1}\left(G^{\theta} \bullet X^{\theta}\right)\right) / 2$.In summary, (3) is equivalent to

$$
\left[\begin{array}{c}
y_{p} \\
y_{c}
\end{array}\right]=\left[\begin{array}{l}
\mathfrak{R}\left\{Q_{M}^{-1}\left(F^{q} \bullet X_{p}^{q}\right)\right\} \\
\mathfrak{I}\left\{Q_{M}^{-1}\left(F^{q} \bullet X_{p}^{q}\right)\right\}
\end{array}\right]+\left[\begin{array}{c}
\mathfrak{I}\left\{Q_{M}^{-1}\left(F^{q} \bullet X_{c}^{q}\right)\right\} \\
\mathfrak{R}\left\{Q_{M}^{-1}\left(F^{q} \bullet X_{c}^{q}\right)\right\}
\end{array}\right]
$$

\subsection{DQDFT parallel filtering algorithm.}

In the high-speed demodulation system with more parallel channels, the computational complexity of the QDFT-based frequency-domain matched filtering algorithm is still large, and the J-module is subdivided into smaller modules to reduce the complexity of the matched filtering algorithm.

Based on the above analysis, a parallel matching filter algorithm with lower complexity is proposed. The algorithm divides the parallel input data into shorter data blocks and inputs them into several $\mathrm{J}$ modules in parallel to obtain the calculation results of the multiple $\mathrm{J}$ modules and carry out the $\mathrm{J}$ module results of the last data block which is retained by the previous data.The corresponding operation, to achieve the purpose of block convolution. The improved frequency domain parallel matching filter algorithm is equivalent to the effect of multiple overlapped data segments matching filtering at the same time, which reduces the computational complexity and improves the ability of real-time processing. In block-based convolutional matched filtering, the data is divided into smaller data blocks, and more $\mathrm{J}$ modules are used. We define the smallest $\mathrm{J}$ module as the unit $\mathrm{J}$ module, the input size is the number of power 2, determined by the filter length. For example, when the filter length $\mathrm{L}$ is equal to 33 , the input to the unit $\mathrm{J}$ module is 64 . If the current data parallel input $\mathrm{n} \mathrm{J}$ module for QDFT matching filtering method, we call nQDFT matching filter algorithm. And $\mathrm{n} J$ modules are 
unit $\mathrm{J}$ module when we called DQDFT matching filtering algorithm.

\section{Complexity analysis and simulation experiment}

In this paper, in order to facilitate the complexity analysis, $\mathrm{M}, \mathrm{m}$ using the power of 4 times the number of hardware used in the widely used base- 4 FFT calculations. The computational complexity of the base-4 FFT can be summarized as follows:

$$
\mu_{M}^{c}=\frac{3}{8} M \log _{2} M ; \quad \alpha_{M}^{c}=M \log _{2} M ; \quad \mu_{2 M}^{\gamma}=\frac{3}{8} M \log _{2} M+2 M ; \quad \alpha_{2 M}^{\gamma}=M \log _{2} M+M
$$

where $\mu_{n}$ and $\alpha_{n}$ mean the number of multiplications and additions for the $\mathrm{n}$-point transform respectively, and the superscript $c$ and $\gamma$ mean the complex and real data respectively.

The total number of multiplications for the traditional overlap-preserving filtering algorithm is $\mu_{2 M}^{\gamma}+M+1+\mu_{2 M}^{\gamma}$, and the number of addition is $\alpha_{2 M}^{\gamma}+\alpha_{2 M}^{\gamma}$. The QDFT algorithm multiplies the input data by the rotation factor (multiplication of $W_{N}^{(3 / 4) n}$ to the n-th input) and uses the standard FFT to complete the calculation. Therefore, the the total number of multiplications for QDFT filtering algorithm is $2 \mu_{M}^{c}+3 M$, and the number of addition is $2 \alpha_{M}^{c}+M$.After the $\mathrm{J}$ module again aliquot of the 2QDFT matching filter method requires $4 \mu_{M / 2}^{c}+3 M$ complex multiplication times, and the number of addition is $4 \alpha_{M / 2}^{c}+M$. It can be seen that, in the block convolutional matching filter, the previous data is divided into smaller data blocks again, and the more J modules are used, the lower the complexity of the algorithm is. The size of the minimum data block depends on the size of the cell $\mathrm{J}$ module and is determined by the filter length.

Table 1 compares the computational complexity of DFT-based overlap-preserving matched filter algorithm and QDFT-based matched filter algorithm and 2QDFT matched filter algorithm.

Table 1 Comparison of algorithm complexity

\begin{tabular}{|c|c|c|}
\hline algorithm & multiplication & addition \\
\hline DFT & $3 \mathrm{M} \log 2 \mathrm{M} / 4+5 \mathrm{M}+1$ & $2 \mathrm{M} \log 2 \mathrm{M}+2 \mathrm{M}$ \\
\hline QDFT & $3 \mathrm{M} \log 2 \mathrm{M} / 4+3 \mathrm{M}$ & $2 \mathrm{M} \log 2 \mathrm{M}+\mathrm{M}$ \\
\hline
\end{tabular}

Table 2 compares the computational complexity (the sum of multiplication and addition) of the three algorithms for several classical segment lengths. As shown in Table 2, the current input data block length is 512, the QDFT-based matched filter algorithm saves $12.6 \%$ of the computation time, and the DQDFT matched filter algorithm saves 38.5\%. The QDFT-based matched filter algorithm saves $10.0 \%$ of the computational complexity while the DQDFT matched filter algorithm saves $51.2 \%$ when the input data block length is 4096 . Therefore, the DQDFT algorithm proposed in this paper can reduce the computational complexity greatly. In practice, it can reduce the running time and hardware resource consumption to improve the demodulation speed.

Table 2 The computational complexity of the three algorithms under typical length

\begin{tabular}{|c|c|c|c|c|c|}
\hline Data length & DFT & QDFT & save(\%) & DQDFT & save(\%) \\
\hline 1024 & 35329 & 31232 & 11.6 & 19968 & 43.4 \\
\hline 2048 & 76289 & 68096 & 10.7 & 39936 & 47.6 \\
\hline 4096 & 163841 & 147456 & 10.0 & 79872 & 51.2 \\
\hline
\end{tabular}




\section{Conclusions}

In this paper, the overlap-preserving method is decomposed. By studying the relationship between QDFT and overlap-preserving method, a low-complexity frequency-domain parallel matching filter algorithm is proposed. Only need to calculate the current data block, than the traditional overlap filter algorithm to preserve the need for shorter FFT length. When the number of parallel paths is large, the algorithm proposed saves more computational complexity than the DFT algorithm. When the segment length reaches 4096, the saving effect reaches more than half. Therefore, the DQDFT matched filtering algorithm can be applied in the high-speed demodulation system and so on.

\section{References}

[1] Ramdani M, Sicard E, Boyer A, et al. The Electromagnetic Compatibility of Integrated Circuits-Past, Present, and Future[J]. IEEE Transactions on Electromagnetic Compatibility, 2009, 51(1):78-100.

[2] SHIHONG D, YAMU H, SAWAN M. A high data rate QPSK demodulator for inductively powered electronics implants[C]//IEEE International Symposium on Circuits and Systems Island of Kos. Greece: IEEE, 2006: 2577-2580.

[3] Chenghuan X, He C, Shunan Z. Design and Implementation of a High-Speed Programmable Polyphase FIR Filter[C]// IEEE 5th ASIC, 2003, 2: 783-787.

[4] Gustafsson O, Dempster A G. On the Use of Multiple Constant Multiplication in Polyphase FIR Filters and Filter Bnaks[C]// IEEE NORSIG04, 2004:53-56.

[5] Srinicasan M,Chen C-C, Gray A. An All-Digital, High Data-Rate Parallel Receiver[R].Jet Propulsion Lab TDA Progress Report, 1997, vol. 42 -131.

[6] Liu Celine, An Jianping, Wang Cuilian, and so on. Low Complexity Frequency Domain Parallel Demodulation Architecture for Joint Symbol Synchronization [J]. Space Electronic Technology, 2013,10 (2): 17-19.

[7] Park S Y, Meher P K. Low-power, high-throughput, and low-area adaptive FIR filter based on distributed arithmetic[J]. IEEE Transations on Circuits and Systems II: Express Bricfs, 2013, 60(6): 346-350. 